\title{
The plantar reflex
}

\section{A study of observer agreement, sensitivity, and observer bias}

\author{
Rui Araújo, MD \\ João Firmino-Machado, MD \\ Pedro Correia, MD \\ Mariana Leitão-Marques, MD \\ João Carvalho, MD \\ Marta Silva, MD \\ Ana Nogueira, MD \\ Carla Nunes, MD
}

\section{Summary}

The utility of the plantar reflex in modern neurology is controversial. We studied the Babinski, Chaddock, and Oppenheim reflexes in terms of intraobserver, interobserver, and intertest agreement; sensitivity; positive predictive value (PPV); and observer bias. Sixty-two patients and 1,984 reflexes were analyzed. Intraobserver and interobserver agreement were weak (median $\kappa<0.4$ ). Intertest agreement was weak (median $\kappa<$ 0.4) for all paired reflexes, although highest for the Babinski/Chaddock (0.30) $(p<0.05)$. There was no evidence of observer bias. Sensitivity was $59.7 \%$ for the Babinski, 55.3\% for the Chaddock, and 30.0\% for the Oppenheim. PPV was $70.3 \%$ for the Babinski, $66.5 \%$ for the Chaddock, and $61.3 \%$ for the Oppen-

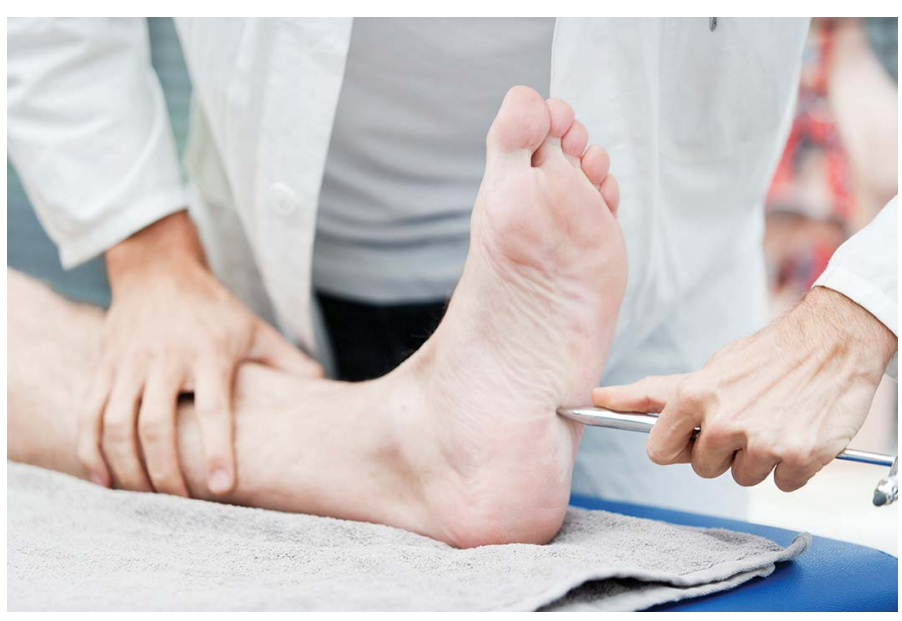
heim. Our results show consistently low observer agreement for the plantar reflex. The Babinski and the Chaddock demonstrated comparable sensitivity and PPV.

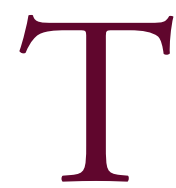

he Babinski sign was first used by Joseph Babinski in $1896^{1}$ to exclude hysteria as a cause of hemiparesis. ${ }^{2}$ Babinski ${ }^{3}$ argued that the plantar reflex was best elicited by stroking the lateral side of the sole. In 1902, Oppenheim described eliciting toe extension by stroking the medial tibia, ${ }^{4}$ whereas Chaddock's 1911 version consisted of stimulating the external inframalleolar region. ${ }^{5}$ Other variants were later described,

Department of Neurology (RA, PC, ML-M, JC, MS, CN) and Department of Internal Medicine (AN), Centro Hospitalar e Universitário de Coimbra (CHUC), Coimbra, Portugal; and Centro Hospitalar do Alto Ave (CHAA) (JF-M), Creixomil, Portugal.

Funding information and disclosures are provided at the end of the article. Full disclosure form information provided by the authors is available with the full text of this article at Neurology.org/cp.

Correspondence to: rmma22@gmail.com 


\section{Globally, our results showed a sensitivity of $59.7 \%$ for the Babinski, 55.3\% for the Chaddock, and 30.0\% for the Oppenheim.}

but only a few have persisted into the 21 st century. ${ }^{6}$ Despite being an integral part of the neurologic examination, ${ }^{7,8}$ the utility of the plantar reflex is controversial., ${ }^{9,10}$ Sensitivity (51\%) and positive predictive value (PPV; 52\%) for pyramidal tract dysfunction (PTD) were recently published for the Babinski reflex, ${ }^{11}$ but values for the Chaddock and Oppenheim variants have not been studied. Interobserver consistency for the Babinski, Chaddock, and Oppenheim reflexes was recently studied: $\kappa$ values ranged from 0.37 to 0.55 , with advantage for the Babinski. ${ }^{6}$ Interobserver agreement was 0.55 for the Babinski, 0.41 for the Chaddock, and 0.37 for the Oppenheim. ${ }^{6}$ Also, there is evidence of a biasing effect of "high value information" (e.g., hemiplegia) in the classification of the plantar reflex. ${ }^{12}$

In this study we investigated the intraobserver and interobserver agreement of the Babinski, Chaddock, and Oppenheim reflexes by examining which pair of tests agreed the most; whether the interpretation of the plantar reflex was influenced by knowing the diagnosis of the patient being tested; and the sensitivity and PPV of the 3 tests for the diagnosis of PTD.

\section{METHODS}

We consecutively recruited patients admitted to our neurology department to obtain a sample size larger than the one calculated on $\mathrm{R}$ program using a study published by Estañol et al. in 1995 as a reference. ${ }^{13}$ Exclusion criteria were agitated patients and patients with lower limb amputation or lower limb cutaneous or soft tissue lesions. Patients with a total or partial anterior circulation infarct ${ }^{14}$ diagnosis with an established lesion on CT or MRI scan were observed by a senior neurologist (with full access to the patient's file) who confirmed the presence of PTD and the side affected. Each patient was observed by 3 different neurologists: observer 1 , in the emergency room, who performed only the Babinski; observer 2, the neurologist in charge of the patient in the medical ward with full access to the patient's file; and observer 3, a "blinded observer" in the medical ward who did not have accessory clinical information and examined the patient while only the legs and feet were visible. Observers were instructed to perform and classify the plantar reflex as explained in a reference textbook on the neurologic examination. ${ }^{7}$ Plantar stimulation for the Babinski began laterally near the heel and was carried up the side of the foot, stopping at the metatarsophalangeal joints or the base of the great toe. The Chaddock was elicited by stimulating the lateral aspect of the foot, beginning under the lateral malleolus and drawing the stimulus from the heel forward to the small toe. The Oppenheim was elicited by dragging the knuckles heavily down the anteromedial surface of the tibia from the infrapatellar region to the ankle. Observers were instructed to classify the reflex as extensor, flexor, or equivocal. Response was considered extensor when the great toe went upward. Fanning of the toes was not considered an extensor response; in case of doubt, observers were instructed to acknowledge the first toe movement of the first stimulation. Response was considered flexor when flexion of the toes occurred after stimulation. Response was considered equivocal when the observer could not decide whether the response was clearly flexor or extensor using these criteria.

Observations performed at the bedside of the patient occurred at "moment T1." The 3 observers classified the plantar reflexes as extensor, flexor, or equivocal. Examinations by observers 2 and 3 were video recorded from the observers' point of view, and only the legs and feet of the patient and the hands and reflex hammer of the observer were recorded. Observations at moment T1 were separated by a maximum of 48 hours, and the authors ensured that no notable clinical changes or de novo neurologic conditions likely to cause 
Table 1 Intraobserver and interobserver agreement for the Babinski, Chaddock, and Oppenheim reflexes

\begin{tabular}{|c|c|c|c|c|c|c|c|}
\hline & \multicolumn{2}{|c|}{ Intraobserver } & \multicolumn{5}{|c|}{ Interobserver } \\
\hline & & $95 \% \mathrm{Cl}$ & $\mathrm{T} 1$ & T2 & $p$ & Global & $95 \% \mathrm{Cl}$ \\
\hline Babinski & 0.37 & $0.25-0.42$ & 0.40 & 0.29 & $<0.05$ & 0.38 & $0.28-0.46$ \\
\hline Chaddock & 0.30 & $0.15-0.41$ & 0.31 & 0.37 & 0.18 & 0.35 & $0.24-0.46$ \\
\hline Oppenheim & 0.35 & $0.15-0.41$ & 0.36 & 0.24 & 0.32 & 0.24 & $0.22-0.49$ \\
\hline
\end{tabular}

different plantar reflex results occurred. Later, after a period of at least 3 months (to eliminate memory bias), the same observers were asked to watch their own examination on a television with no sound and to reclassify the plantar reflex. This was termed "moment T2."

Observers were instructed to use the sharp end of their reflex hammer to elicit the Babinski and Chaddock reflexes and to repeat the procedure as many times as necessary. A total of 10 neurologists were involved (2 first-year residents, 2 third-year residents, 2 fifth-year residents, and 4 senior neurologists).

Agreement was defined as the same classification by 2 observers. Intraobserver agreement was tested by comparing the responses of observers 2 and 3 between T1 and T2. Interobserver agreement was tested by comparing the responses of different observers at the same moment in time. Intertest agreement was assessed by comparing the responses of groups of 2 . Observer bias was studied by comparing the responses of blinded and nonblinded observers (in particular, the interobserver agreement for observers 2 and 3 at moment $\mathrm{T} 1$ and the intraobserver agreement for observer 2). Sensitivity and PPV were calculated using the extensor response in patients with established PTD. Specificity and negative predictive value were not calculated because of uncertainty regarding the existence of PTD in the rest of the patients. $\kappa$ statistic was used to assess agreement, as described by Landis and $\operatorname{Koch}^{15}$ ( $\kappa$ median less than $0.41=$ weak agreement, $0.41-0.6=$ moderate agreement, $0.61-0.8=$ substantial agreement, and $0.81-1.0=$ excellent agreement). Wilcoxon test (exact) and Kruskal-Wallis test for the paired data (significance level of 0.05) were used for differences between the results obtained at different time points and between reflexes. A 95\% confidence interval (CI) was calculated for all tests. The use of nonparametric tests was based on the analysis of data distribution using the ShapiroWilk test. Standards for Reporting of Diagnostic Accuracy criteria were considered in the study design and reporting of results. ${ }^{16}$

\section{Standard protocol approvals, registrations, and patient consents}

Informed consent was given by the patients or their legal representatives. Patient privacy and data confidentiality were guaranteed. Ethics approval for this project was obtained by the local ethics commission.

\section{RESULTS}

Sixty-two patients were analyzed: 38 (62\%) with PTD, 5 with cerebral vascular disease without PTD (2 with lacunar anterior cerebral infarction, 2 with posterior cerebral infarction, 1 with TIA), 5 with a form of parkinsonism (3 with Parkinson disease, 2 with multiple system atrophy), 4 with dementia (3 with Alzheimer disease, 1 with frontotemporal dementia), 4 with multiple sclerosis, 2 with encephalitis, 2 with status epilepticus, 1 with hydrocephalus, and 1 with myasthenia gravis. Eighteen patients were excluded ( 11 were agitated, 6 had significant lesions in the lower limbs, and 1 had lower limb amputation). Each patient was tested 32 times between 3 observers (14 times for T1 and 18 times for T2, because observer 1 in T1 performed only the 


\begin{tabular}{|c|c|c|c|c|c|c|c|}
\hline \multirow[t]{3}{*}{ Table 2} & $\begin{array}{l}\text { y and } \\
\text { midal }\end{array}$ & $\begin{array}{l}\text { itive pr } \\
\text { t dysf }\end{array}$ & $\begin{array}{l}\text { tive va } \\
\text { ion }\end{array}$ & PV) of a & xtenso & ponse & atients \\
\hline & \multicolumn{4}{|c|}{ Sensitivity } & \multicolumn{3}{|l|}{ PPV } \\
\hline & $\mathrm{T} 1$ & T2 & $p$ & Global & $\mathrm{T} 1$ & T2 & Global \\
\hline Babinski & 58.8 & 60.5 & 0.18 & 59.7 & 72.4 & 68.3 & 70.3 \\
\hline Chaddock & 64.5 & 49.1 & $<0.05$ & 55.3 & 75.4 & 60.2 & 66.5 \\
\hline Oppenheim & 32.9 & 28.1 & 0.32 & 30.0 & 58.1 & 64.4 & 61.3 \\
\hline
\end{tabular}

Babinski). The Babinski was analyzed 456 times in the PTD group and 744 times for all patients. The Chaddock and Oppenheim were analyzed 380 times for the PTD group and 620 times in total. A total of 1,984 reflexes were analyzed, of which 1,216 were in the PTD subgroup. Ages of the patients ranged from 32 to 98 years (mean 72.6 ); $47 \%$ were male and $53 \%$ were female. Fifteen patients (24.2\%) had type 2 diabetes mellitus, 3 patients (4.8\%) had documented polyneuropathy, and 2 patients $(3.2 \%)$ had radicular pain secondary to lumbar disk hernia. No other medical conditions capable of influencing the plantar reflex were identified.

All observers used the sharp end of their reflex hammer to elicit a nociceptive plantar reflex. Nine neurologists used a Queen Square-type reflex hammer and one used a Babinski-type reflex hammer.

\section{Intraobserver and interobserver agreement}

Intraobserver agreement was weak for all tests. The Babinski scored 0.37 (95\% CI 0.25-0.42), the Oppenheim 0.35 (95\% CI 0.15-0.41), and the Chaddock 0.30 (95\% CI 0.15-0.41), with no significant difference between them $(p=0.90)$. Interobserver agreement was also weak (median $\kappa$ values were $0.31-0.40$ for $\mathrm{T} 1,0.24-0.37$ for $\mathrm{T} 2$, and $0.24-0.38$ overall), with no difference between tests. There was a significant difference in test agreement between T1 (0.40) and T2 (0.29) for the Babinski (table 1).

\section{Intertest agreement}

Intertest agreement was weak. The Babinski/Chaddock scored 0.30 (95\% CI 0.22-0.41), the Babinski/Oppenheim 0.20 (95\% CI 0.12-0.28), and the Chaddock/Oppenheim 0.23 (95\% CI 0.07-0.31), with a difference between Babinski/Oppenheim and the other 2 pairs $(p<0.05)$.

\section{Sensitivity and PPV}

The sensitivity of the plantar reflex for PTD at T1 was highest for the Chaddock (64.5\%), followed by the Babinski (58.8\%) and the Oppenheim (32.9\%). For T2, the Babinski scored $60.5 \%$, the Chaddock 49.1\%, and the Oppenheim 28.1\%. Globally, our results showed a

Table 3 Intraobserver and interobserver agreement for the 3 observers

\begin{tabular}{|c|c|c|c|c|c|}
\hline $\begin{array}{l}\text { Interobserver } \\
\text { agreement (T1) }\end{array}$ & Median $\kappa$ & $95 \% \mathrm{Cl}$ & $\begin{array}{l}\text { Intraobserver } \\
\text { agreement }\end{array}$ & Median к & $95 \% \mathrm{Cl}$ \\
\hline Pair of observers & & & Pair of observers & & \\
\hline $1 / 2$ & 0.24 & $0.06-0.46$ & $1 / 1$ & 0.44 & $0.44-0.44$ \\
\hline $1 / 3$ & 0.22 & $0.04-0.38$ & $2 / 2$ & 0.30 & $0.15-0.45$ \\
\hline $2 / 3$ & 0.32 & $0.22-0.46$ & $3 / 3$ & 0.30 & $0.10-0.42$ \\
\hline p Value & 0.09 & & & 0.24 & \\
\hline
\end{tabular}




\section{While recent studies have calculated the} sensitivity and positive predictive value of the Babinski sign, we also put the Chaddock and Oppenheim variants to the test.

sensitivity of $59.7 \%$ for the Babinski, $55.3 \%$ for the Chaddock, and $30.0 \%$ for the Oppenheim. There was a difference for extensor responses for the Chaddock between T1 and T2 $(p<0.05)$. At T1, PPV was highest for the Chaddock $(75.4 \%)$, followed by the Babinski (72.4\%) and the Oppenheim (58.1\%). For the video evaluation (T2), the Babinski had the highest PPV (68.3\%), followed by the Oppenheim (64.4\%) and the Chaddock (60.2\%). Globally, the Babinski showed a 70.3\% PPV for PTD, followed by the Chaddock (66.5\%) and the Oppenheim (61.3\%) (table 2).

\section{Observer bias}

Interobserver agreement at T1 for observers 2 ("open") and 3 ("blinded") was 0.32 , the highest of all observer combinations. Intraobserver agreement for observer 2 was 0.30 ; median $\kappa$ value for observer 1 was 0.44 and for observer 3 was 0.30 (table 3).

\section{DISCUSSION}

A review of previous studies on the plantar reflex is provided in table 4 .

\section{Intraobserver agreement}

We found a weak median $\mathrm{\kappa}$ value $(<0.4)$ for all 3 tests, with no significant difference between the groups. These results do not differ greatly from the results of Singerman and $\mathrm{Lee}^{6}$ (0.35 for the Babinski, 0.52 for the Chaddock, and 0.49 for the Oppenheim), even though we included 62 patients and Singerman and Lee included only 6 patients observed twice. Maher et al. ${ }^{17}$ Isaza Jaramillo et al., ${ }^{11}$ and Estañol et al. ${ }^{13}$ report higher intraobserver agreement for the Babinski $(0.60,0.57$, and 0.98 , respectively). A possible explanation for this is that our study examiners were allowed to classify the reflex into 3 different categories, whereas in the study by Isaza Jaramillo et al. ${ }^{11}$ a neutral response was considered the same as flexor and in the study by Maher et al. ${ }^{17}$ a neutral response was not accepted. In our study, the Chaddock and Oppenheim do not seem to perform differently from the Babinski.

\section{Interobserver agreement}

We also found weak interobserver agreement (median $\kappa<0.4$ ), with no significant difference between tests. Previous studies have shown similar and higher values. Miller and Johnson ${ }^{10}$ reported 0.30 for the Babinski, and Singerman and $\mathrm{Lee}^{6}$ reported 0.55 for the Babinski and less for the other variants (Chaddock 0.41 and Oppenheim 0.37). Isaza Jaramillo et al. ${ }^{11}$ showed that the accuracy of the Babinski sign to diagnose PTD was similar to the area under a receiver operating characteristic curve, with a reliability of 0.73 . Again, these discrepancies could be explained by the fact that our study observers were allowed to classify the reflex in 3 ways rather than 2 .

\section{Intertest agreement}

Intertest agreement was weak (median $\kappa<0.4$ ) for all paired reflexes, although highest for the Babinski/Chaddock $(0.30)(p<0.05)$. This result, although lower than that found by Singerman and Lee $(0.57),{ }^{6}$ shows that the Babinski/Chaddock combination appears to be the most agreed upon. The globally lower agreement in our study can be explained by the flexor/extensor dichotomy previously outlined. 
Table 4 Review of the literature regarding the study of the Babinski sign and its variants

\begin{tabular}{|c|c|c|c|c|c|c|c|c|}
\hline Ref. & $\begin{array}{l}\text { No. of } \\
\text { patients }\end{array}$ & Tests & $\begin{array}{l}\text { No. of } \\
\text { tests }\end{array}$ & $\begin{array}{l}\text { Sensitivity } \\
\text { (PTD) }\end{array}$ & $\begin{array}{l}\text { PPV } \\
\text { (PTD) }\end{array}$ & $\begin{array}{l}\text { Intraobserver } \\
\text { agreement }\end{array}$ & $\begin{array}{l}\text { Interobserver } \\
\text { agreement }\end{array}$ & $\begin{array}{l}\text { Intertest } \\
\text { agreement }\end{array}$ \\
\hline 17 & 12 & $\mathrm{Bab}$ & 192 & NA & NA & 0.60 & 0.17 & NA \\
\hline 13 & 200 & Bab & $800^{a}$ & $84 \%$ & $99 \%$ & 0.98 & NA & NA \\
\hline 10 & 10 & $\begin{array}{l}\text { Bab, foot } \\
\text { tapping }\end{array}$ & $\begin{array}{l}200 \\
(\mathrm{Bab})\end{array}$ & $40 \%-65 \%$ & NA & NA & 0.30 & NA \\
\hline 6 & 34 & $\begin{array}{l}\text { Bab, Cha, } \\
\text { Opp, Gord }\end{array}$ & 320 & NA & NA & $\begin{array}{l}\text { Bab 0.35, } \\
\text { Cha } 0.52, \\
\text { Opp } 0.49^{b}\end{array}$ & $\begin{array}{l}\text { Bab 0.55, } \\
\text { Cha 0.41, } \\
\text { Opp } 0.37\end{array}$ & $\begin{array}{l}\text { Bab/Cha 0.57, } \\
\text { Bab/Opp 0.31, } \\
\text { Cha/Opp 0.31 }\end{array}$ \\
\hline 11 & 107 & $\mathrm{Bab}$ & 856 & $51 \%$ & $52 \%$ & 0.57 & 0.73 & NA \\
\hline $\begin{array}{l}\text { Present } \\
\text { study }\end{array}$ & 62 & $\begin{array}{l}\text { Bab, Cha, } \\
\text { Opp }\end{array}$ & 1,984 & $\begin{array}{l}\text { Bab } 60 \%, \\
\text { Cha 55\%, } \\
\text { Opp 30\%c }\end{array}$ & $\begin{array}{l}\text { Bab } 70 \% \text {, } \\
\text { Cha } 67 \% \text {, } \\
\text { Opp } 61 \%{ }^{c}\end{array}$ & $\begin{array}{l}\text { Bab 0.37, } \\
\text { Cha 0.30, } \\
\text { Opp } 0.35\end{array}$ & $\begin{array}{l}\text { Bab 0.38, } \\
\text { Cha 0.35, } \\
\text { Opp 0.24 }\end{array}$ & $\begin{array}{l}\text { Bab/Cha 0.30, } \\
\text { Bab/Opp 0.20, } \\
\text { Cha/Opp } 0.23\end{array}$ \\
\hline
\end{tabular}

Abbreviations: $\mathrm{Bab}=$ Babinski; $\mathrm{Cha}=$ Chaddock; Gord = Gordon; NA = not available; Opp = Oppenheim; PPV = positive predictive value; PTD = pyramidal tract dysfunction

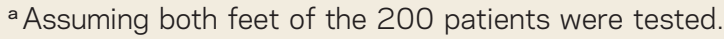

${ }^{b}$ Only 6 patients for intraobserver analysis.

${ }^{c}$ Only 32 patients for sensitivity and PPV analysis.

\section{Sensitivity and PPV}

Our results show a sensitivity for PTD of approximately $60 \%$ for the Babinski and 55\% for the Chaddock. The Babinski result from our study is not very different from that found by Miller and Johnson ${ }^{10}$ or Isaza Jaramillo et al. ${ }^{11}$ While previous studies on the plantar reflex provided information regarding the accuracy of the Babinski sign for acute PTD diagnosis, we additionally provide insight into the diagnostic value of the Chaddock and Oppenheim.

Sensitivity for acute PTD in the actual patient setting was highest for the Chaddock (64.8\%). The Oppenheim variant had approximately half of the sensitivity of the other variants and had the lowest PPV of all tests. PPV in the actual patient setting was also highest for the Chaddock (75.4\%). Overall, PPV was 70\% for the Babinski and 66\% for the Chaddock; this is higher than in Isaza Jaramillo et al., ${ }^{11}$ in which the PPV for the Babinski was approximately $50 \%$. Our results suggest that the Chaddock might perform even better than the Babinski in real patients. However, this must be interpreted in light of the consistently and globally low observer agreement reported.

\section{Observer bias}

The presence of an observer bias in the classification of the plantar reflex had not been addressed since the pivotal study by Van Gijn and Bonke in $1972 .{ }^{12}$ In our study, agreement between "open" (observer 2) and "blinded" (observer 3) examinations at moment T1 was 0.32 , but no significant difference was found between the groups. If observer bias existed, one would expect the median kappa agreement to be lowest between Observer 2 and 3. Also, the intraobserver agreement for observer 2 was 0.30 , again with no significant difference. These results do not confirm observer bias, conflicting with the results of Van Gijn and Bonke, ${ }^{12}$ but our results must be cautiously interpreted in light of globally low agreement.

\section{CONCLUSIONS}

Our study brings new insight into the plantar reflex by analyzing a robust sample of reflexes. We allowed observers to classify the reflex in 3 ways (including equivocal), which we feel best translates into clinical practice and probably accounts for the globally lower agreement values. We fully agree with Professor William Landau's statement: "Plantar reflex patterns are not restricted to only two up-or-down, chocolate-or-vanilla flavors," 18 and we feel our study 
addresses this issue. While recent studies have calculated the sensitivity and PPV of the Babinski sign, we also put the Chaddock and Oppenheim variants to the test. Our results show that the Chaddock can rival the Babinski in terms of sensitivity and PPV, constituting a valid and probably underused alternative. Our study included only vascular models of PTD, which could limit generalization of results regarding sensitivity and PPV to other models of PTD. As previous studies have suggested, intraobserver and interobserver agreement for all variations of the plantar reflex are consistently low. Our results do not suggest the presence of observer bias. The Babinski and Chaddock were also the variants that agreed the most between themselves, in concordance with previous literature. Despite observer "disagreement," we feel the plantar reflex, particularly when performed and interpreted alongside other essential features of the neurologic examination (such as tonus, motor power, and reflexes), is still very important in the bedside diagnosis of PTD.

\section{REFERENCES}

1. Babinski J. Sur le refléxe cutané plaintaire dans certraines affections organiques du systeme nerveux central. C R Soc Biol 1896;48:207-208.

2. Babinski J. Du phénomène des orteils et de sa valeur sémiologique. Sem méd 1898;18:321-322.

3. Babinski J. Relachement des muscles dans l'hémiplégie organique. C R de la Soc Biol 1896;48: 471-472.

4. Oppenheim H. Zur pathologie der hautreflexe an den unteren extremitäten. Mschr Psychiatr Neurol 1902;12:518-530.

5. Chaddock C. The external malleolar sign. Interstate Med J 1911;18:1026-1038.

6. Singerman J, Lee L. Consistency of the Babinski reflex and its variants. Eur J Neurol 2008;15:960-964.

7. Campbell WW. Pathologic reflexes. In: Campbell WW. DeJong's The Neurologic Examination, 7th ed. Philadelphia, PA: Wolters Kluwer Health/Lippincott, Williams \& Wilkins; 2013:583-597.

8. Biller J, Gruener G, Brazis P. DeMyer's The Neurologic Examination: A Programmed Text, 6th ed. New York, NY: The McGraw-Hill Companies; 2011.

9. Rayner PHW. The Babinski sign. Eliciting the sign brings out doctors' masochistic tendencies. BMJ 1997;314:374.

10. Miller TM, Johnson SC. Should the Babinski sign be part of the routine neurologic examination? Neurology 2005;65:1165-1168.

11. Isaza Jaramillo SP, Uribe Uribe CS, García Jimenez FA, Cornejo-Ochoa W, Alvarez Restrepo JF, Román GC. Accuracy of the Babinski sign in the identification of pyramidal tract dysfunction. J Neurol Sci 2014;343:66-68.

12. Van Gijn J, Bonke B. Interpretation of plantar reflexes: biasing effects of other signs and symptoms. J Neurol Neurosurg Psychiatry 1977;40:787-789.

13. Estañol B, Jiménez-Gil FJ, Cárdenas E, Corona T. Babinski’s sign: statistical validity of a classic sign in medicine. Neurologia 1995;10:307.

14. Bamford J, Sandercock P, Dennis M, Burn J, Warlow C. Classification and natural history of clinically identifiable subtypes of clinical infarction. Lancet 1991;337:1521-1526.

15. Landis JR, Koch CG. The measurement of observer agreement for categorical data. Biometrics 1977; 33:159-174.

16. Bossuyt P, Reitsma J, Bruns D, et al; Standards for Reporting of Diagnostic Accuracy. The STARD statement for reporting studies of diagnostic accuracy: explanation and elaboration. Ann Intern Med 2003;138:W1-W12.

17. Maher J, Reilly M, Daly L, Hutchinson M. Plantar power: reproducibility of the plantar response. BMJ 1992;304:482.

18. Landau W. Plantar reflex amusement: misuse, ruse, disuse, and abuse. Neurology 2005;65:1150-1151.

\section{ACKNOWLEDGMENT}

The authors thank Dr. João Lemos (Neurology Department, CHUC), Dr. João Sargento-Freitas (Neurology Department, CHUC), and Dr. Paulo Coelho (Neurology Department, ULS-Matosinhos) for helpful suggestions.

\section{AUTHOR CONTRIBUTIONS:}

Rui Araújo took the initiative for the paper, designed most of the study model, and provided significant input into early and final versions of the manuscript. João Firmino-Machado led the statistical analysis and 
provided significant input into the final version of the manuscript. Pedro Correia provided significant input into early versions of the manuscript and participated as an examiner in the study. Mariana Leitão-Marques provided significant input into early versions of the manuscript, aided in the study design, and participated as an examiner. João Carvalho provided significant input into early and final versions of the manuscript and participated as an examiner. Marta Silva participated as an examiner, participated in the interpretation of the statistical analysis, and provided significant input into the final version of the manuscript. Ana Nogueira contributed to patient selection, provided input into early versions of the manuscript, and filmed most of the examinations. Carla Nunes oversaw the entire project, helped in the study design, provided significant input into early and final versions of the manuscript, and is the guarantor of the article.

\section{STUDY FUNDING}

No targeted funding reported.

\section{DISCLOSURES}

R. Araújo has received funding for travel from Teva Neuroscience and Boehringer-Ingelheim. J. Firmino-Machado and P. Correia report no disclosures. M. Leitão-Marques has received speaker honoraria from Tecnifar. J. Carvalho has received funding for travel from Krka Pharmaceuticals and for a registration fee from Servier. M. Silva has received funding for travel from Teva and Genzyme. A. Nogueira reports no disclosures. C. Nunes serves on a scientific advisory board for Biogen and received funding for travel from Sanofi. Full disclosure form information provided by the authors is available with the full text of this article at Neurology.org/cp.

\section{Related articles from AAN physician and patient resources}

\section{Neurology ${ }^{\circledR} \quad$ Neurology.org}

Plantar reflex amusement: Misuse, ruse, disuse, and abuse October 25, 2005;65:1150-1151.

\section{Continuum ${ }^{\circledR}$ - ContinuumJournal.com}

Clinical Approach to Peripheral Neuropathy: Anatomic Localization and Diagnostic Testing February 2012;18:13-38. 